\title{
SCENARIO OF ACCELERATED UNIVERSE EXPANSION UNDER EXPOSURE TO ENTROPIC FORCES RELATED TO WITH THE ENTROPIES OF BARROW AND TSALLIS-CIRTO
}

\author{
A.V. KOLESNICHENKO ${ }^{1 *}$, M.YA. MAROV ${ }^{2}$ \\ ${ }^{1}$ Keldysh Institute of Applied Mathematics, Russian Academy of Science \\ ${ }^{2}$ Institute of Geochemistry and Analytical Chemistry. V.I. Vernadsky \\ Russian Academy of Science \\ "Corresponding author. E-mail: kolesn@keldysh.ru
}

\section{DOI: 10.20948/mathmontis-2021-50-8}

Summary. In the work within the framework of "entropic cosmology", the scenario of the cosmological accelerated expansion of a flat, homogeneous and isotropic Universe under the influence of entropic forces is considered without the concept of dark energy- a hypothetical medium with negative pressure. Assuming that the horizon of the Universe has its own temperature and entropy, which arises during the holographic storage of information on the screen of the horizon surface, the entropy models of the Universe associated with the Bekenstein-Hawking entropy and the non-extensive Barrow and Tsallis-Cirto entropies are considered. The modified equations of acceleration and continuity of Friedman with governing power terms having an entropic nature are derived both within the framework of Einstein's general theory of relativity and on the basis of a thermodynamic approach that allows modeling the non-adiabatic evolution of the Universe. At the same time, models based on nonextensive entropies predict the existence of both a decelerating and accelerating Universe.

\section{INTRODUCTION}

In the last years of the twentieth century (1998), an unexpected discovery was made in cosmology related to the accelerating expansion of the Universe. Currently, this fact has been confirmed by a huge number of observational data and numerous cosmological experiments concerning the microwave background, large-scale structure, and other dimensions of the Universe (see, for example, [1-2]). In this regard, modern cosmological concepts are fully consistent with the Friedman-Robertson-Walker model of a homogeneous, isotropic, and almost flat (infinite) and open Universe, continuously expanding with acceleration [3-5].

Despite the growing amount of observational evidence for the existence of an accelerated expansion of the Universe, its nature and fundamental origin are still an unresolved issue. As it is know, the ratio of ordinary (baryonic) matter, dark matter and dark energy is approximately. $1: 10: 25$. Consequently, the evolution of the Universe is completely dominated by cold dark matter and dark energy - the so-called cosmic vacuum [6-7], the energy density of which is currently associated with the cosmological constant $\Lambda=1.1 \times 10^{-56} \mathrm{sm}^{-2}$.

2010 Mathematics Subject Classification: $85 \mathrm{~A} 35,91 \mathrm{~B} 50,82 \mathrm{C} 40$.

Key words and Phrases: Accelerated expansion of the Universe, Entropy cosmology, Barrow, Bekenstein- Hawking and Tsallis-Cirto entropies. 
The constant $\Lambda$ determines antigravity in the Einstein modified general theory of relativity (GR) by Einstein [4].

The cosmological vacuum has everywhere and always constant positive density $\rho_{v}=\Lambda c^{2} / 8 \pi G$ and negative pressure $P_{v}=-c^{2} \rho_{v}$. According to Friedman's cosmology of a homogeneous and isotropic universe, gravitation is created not only by the density of the material medium, but also by its pressure in combination $\rho+3 P / c^{2}$. The vacuum causes antigravity precisely because its effective gravitating energy $\rho_{G}=\rho_{v}+3 P_{v} / c^{2}=-2 \rho_{v}$ is negative at positive density. Since the density of the vacuum (dark energy) exceeds the total density of all other types of cosmic energy, then antigravitation is stronger than gravitation. Under this condition, the cosmological expansion must occur with acceleration [8]. Thus, the cosmological accelerated expansion of the Universe is completely determined by the acting in parallel gravitational and antigravitational forces described by the modified general relativity approach.

It should be noted, however, that at present there are a number theories in support that of gravity are called. In contrast to the Standard Model, which combines three interactions in nature but gravity, the "Theory of Everything" (or M-theory), unifies all forces and particles in nature, but it not fully complies with General relativity [9].

Among the many scenarios for the accelerated expansion of the Universe, the so-called "entropic cosmology" has recently attracted much attention, according to which gravity is perceived as a kind of force associated with a change in entropy. The concept of the cosmological entropic force was proposed by the Dutch physicist ("string theorist") Eric Verlinde, who in his article [10] developed a rather "crazy" theory, according to which the phenomenon of gravity is explained through entropy, i.e. the force of gravity is inherently thermodynamic in origin [11], 2010). In this work, the author argues that the central concept necessary for the emergence of gravity is information (more precisely, the amount of information associated with matter and its distribution) in terms of entropy. The most important assumption of the theory is that information associated with a certain region of space obeys the holographic principle (see, for example, [12] and relies heavily on the physics of black holes [13-14].

In the cited article, it was shown that within the holographic principle of the formation of space $^{\mathrm{i})}$ gravity inevitably arises, which is identified with the entropy force caused by changes in information ${ }^{\text {ii)}}$, associated with the growth of the area occupied by material bodies. According to the holographic picture of the world, entropy is stored on holographic screens, and space appears between two similar screens. With this approach, the gravitational force in space is determined by the entropy gradient, or the so-called entropic force.

Nearly the same time, within the framework of the Verlinde hypothesis, Easson et al. [15] developed a heuristic theory of the accelerated expansion of the Universe, based on the entropic force. Authors of that work have demonstrated that accelerated expansion is an inevitable consequence of an increase in entropy associated with the storage of holographic information on a surface screen located on the event horizon (space-time region) of the Universe. As a result, with this approach, the progress in physical understanding of the process of accelerated expansion of

\footnotetext{
i) Here, holography refers to information about the Universe encoded on a screen, which is interpreted as a twodimensional surface of the Universe.

ii) According to the holographic principle, the growth of information associated with an increase in the surface of the Universe occupied by material bodies leads to an increase in entropy; hence the emergence of a gradient of entropy (entropy force) directed against the increase in the radius of the specified surface area. And this is gravity.
} 
the Universe was achieved based on entropic forces, without the concept of dark energy - hypothetical medium with negative pressure.

In other words, contrary to the widespread explanation of the observed accelerated expansion of the Universe, which appears in the presence of a driving force (in the Friedman equations) due to dark energy, an alternative interpretation of such a force was proposed - an entropic force. The latter was associated with the entropy and temperature of the horizon of the Universe, which arise when storing information on the screen of the surface of the horizon.

Finally, in a number of subsequent works (see, for example, [16-29]) devoted to entropic cosmology, the scenario of the accelerated expansion of the Universe under the influence of entropic forces of various nature was discussed, proceeding from the idea that the horizon of the Universe (like the event horizon of a black hole) has its own entropy and temperature. In all these studies, along with the de Sitter temperature [30], various entropy entities were used (in particular, the Bekenstein-Hawking entropy [13], the non-extensive Tsallis-Cirto entropy [31], the modified (equally-distributed) entropy Renyi [32] and others). Instead of the cosmological constant in the equations of Einstein's general theory of relativity, an additional so-called governing term was added, associated with the entropy and temperature of the event horizon of the Universe. Using modified Friedman equations, it was shown that such models explain the current accelerating expansion of the Universe and they are in good agreement with the data on supernovae. Let us note that the cosmological acceleration found in this case (considered as a consequence of the entropic force) turns out to be relatively small (of the order of the Hubble constant), in contrast to the huge value of accelerated expansion, which is confusing to most cosmologists, predicted by quantum field theory in combination with general relativity ${ }^{\text {iii) }}$.

Thus, the study of the influence of entropic forces on the accelerated expansion of the Universe is of interest, since due to the anti-gravitational action, it is these forces that can play the role of mysterious dark energy both in the form of a cosmological constant and in the form of scalar fields [33]. In entropic cosmology, it is assumed that the horizon of the Universe has associated temperature and entropy due to information stored on the surface of the event horizon holographically.

Here we concern some elementary considerations intended to show how the entropy force, which has a thermodynamic nature, is related to the entropy of a large body. For this, we use the second law of thermodynamics for a macroscopic body, in the form of the Gibbs relation $d E=T d S-P d V$. Since for a very large body with a change in its volume (due to the displacement of the boundary $d r$ ), the surface area $A$ and internal energy $E$ practically do not change, then one can write $0=T d S-P(A d r)$. Hence, it follows that if the entropy changes due to increase of radius of the volume, then the force $F_{S}=P A=T d S / d r$ arises. Since the space-timedependent entropy (evolving in time and reaching a maximum in the final thermal state), expands in space, its gradient appears, which is interpreted as an entropic force.

\footnotetext{
iii) The identification of the cosmological constant with the vacuum energy does not allow, unfortunately, to penetrate into the essence of dark energy and leads to a still unsolvable problem, which consists in the fact that the observed value of the dark energy density $\rho_{\Lambda_{a b s}} \approx\left(10^{-3} \mathrm{eV}\right)^{4}$ and its theoretically predicted value $\rho_{\Lambda_{t h}} \approx 10^{18}(\mathrm{GeV})^{4}$ differ by 120 orders of magnitude (here, $V=V(\varphi)$ the potential of the scalar fields $\varphi$ (inflaton) [4].
} 
In the presented work, which is related to modeling the accelerated expansion of a flat, homogeneous and isotropic Universe, modified Friedman equations are obtained, in which instead of the cosmological constant there appears an additional control term (driving force) associated with changes in entropy and temperature on the Hubble horizon of the Universe. The surface area of the Universe is a key characteristic that determines its entropy and information content. Along with the traditional Bekenstein-Hawking entropy [15], which is proportional to the area of the Hubble horizon, we also incorporate the non-additive Tsallis-Cirto entropy [31], which is proportional to the horizon volume, and the non-additive entropy of Barrow [34-36], taking into account the fractal structure of the Hubble horizon. For these entropies, modified Friedman equations have been constructed to explain the cosmological expansion of the Universe without dark energy. In this case, the corresponding entropic forces predetermine both deceleration and/or accelerated expansion of the Universe. It is important to note that the construction of new models of the evolution of the Universe is carried out on the basis of the recently introduced nonadditive Barrow entropy, which is a new holographic model of entropy associated with the modification of the horizon of the Universe surface due to quantum gravitational effects.

\section{SOME ELEMENTS OF CLASSICAL COSMOLOGY}

\subsection{Gravitational field equations}

First, we will consider a flat evolutionary model of the Universe, which is infinite in space, homogeneous, isotropic and expanding. In this case, the Universe is modeled by some cosmological fluid, the particles of which are galaxies. At this level of large-scale averaging, the structure of the Universe is symmetric and has no singularities. In classical cosmology, models of the evolving Universe are constructed on the basis of Einstein's equations of general relativity (see, for example, [4, 33, 37].

The expansion of the Universe is governed by the equations of the gravitational field, which have the following general form $[4,8]$ :

$$
R_{\mu \nu}-\frac{1}{2} g_{\mu \nu} R-\Lambda(t) g_{\mu \nu}=\kappa T_{\mu \nu}
$$

Here $d s^{2}=g_{\mu v} d x^{\mu} d x^{\nu}$ - four-dimensional space-time interval in general relativity, $g_{\mu \nu}$ - metric tensor, $g_{\mu \nu} g^{\nu \beta}=\delta_{\mu}^{\beta} ; R_{\mu \nu}=g^{\alpha \beta} R_{\mu \nu \alpha \beta}-$ Ricci tensor; $R_{\mu \nu \alpha \beta}-$ the Riemann-Christoffel tensor, composed of the products of the first derivatives $\left(\partial g_{p v} / \partial x_{\alpha}\right) \cdot\left(\partial g_{\beta \mu} / \partial x_{p}\right)$ and the second derivatives $\partial^{2} g_{\mu v} / \partial x_{\alpha} \partial x_{\beta}$ of the metric tensor; $R=g^{\mu v} R_{\mu \nu}$ - scalar curvature of fourdimensional space; $\kappa=8 \pi G / c^{4}-$ Einstein gravitational constant; $T_{\mu \nu}$ - the energy-momentum tensor, which plays the role of the source of the gravitational field; $c$ - speed of light in vacuum, $\Lambda$ - the cosmological "constant" introduced by Einstein, which can often be omitted; $G$ - gravitational constant. 
In flat hyperspace, the space-time linear interval has the form $d s^{2}=c^{2} d t^{2}-a(t)^{2}\left(d x^{2}+d y^{2}+d z^{2}\right)$, which corresponds to the metric tensor with Galilean components $^{\text {iv) }}$

$$
g_{00}=c^{2} ; g_{11}=g_{22}=g_{33}=-a(t)^{2} ; g_{\mu \nu}=0 \text { at } \mu \neq v ; g_{\mu \nu}=g^{\mu \nu} \text {, }
$$

where $t$ - the space time coordinate; $a(t)$ - expansion coefficient (Robertson-Walker scale factor [4]. For the case of an ideal cosmological fluid ${ }^{\mathrm{v})}$ the energy-momentum tensor in a locally inertial Cartesian coordinate system has the form $T_{\mu \nu}=\left(\rho c^{2}+P\right) u_{\mu} u_{v}+P g_{\mu \nu}$, where $\rho=\rho(t)$, $P=P(\rho)$ are, respectively, the density and scalar pressure of the cosmological fluid (including matter and radiation) at the moment of time $t$. Here a four-dimensional velocity $u_{\mu}=\partial x_{\mu} / \partial s$ is introduced, which is determined by the condition that in the accompanying locally inertial Cartesian coordinate system its components are equal $u_{0}=1$ and $u_{\mu \neq 0}=0$. Thus, at rest, the tensor components $T_{\mu \nu}$ have the following form [3]:

$$
T_{00}=\rho c^{2} ; \quad T_{11}=T_{22}=T_{33}=-P ; \quad T_{\mu \nu}=0 \text { npu } \mu \neq v .
$$

Note that in a flat model of the Universe, the three-dimensional curvature is zero, but the fourdimensional space remains curved.

\subsection{Friedman's cosmological model}

Let us consider Friedman's standard model for a flat open universe ${ }^{\mathrm{vi})}$.

From Einstein's equations (1) under the above assumptions ${ }^{\text {vii) }}$ two Friedman equations for the scale factor $a(t)$ follow [3]

$$
\left(\frac{a, t}{a}\right)^{2} \equiv H(t)^{2}=\frac{8 \pi G}{3} \rho(t)+\underset{\text { omitted }}{\Lambda / 3},
$$

\footnotetext{
iv) Almost all modern cosmology is based on this Robertson-Walker metric.

v) An ideal fluid is defined as a medium for which at each point there is a locally inertial Cartesian frame of reference moving with the fluid, in which the fluid itself looks the same in all directions.

vi) Space is flat only if the ratio $\Omega:=\rho / \rho_{c r} \cong 1$, where $\rho_{c r}:=3 H^{2} / 8 \pi G$ is the critical mass density (matter + radiation), $\rho_{c r}=10^{-29} \mathrm{~g} / \mathrm{sm}^{3}$. According to modern observational data, the value $\Omega=1.02 \pm 0.02$.

vii) Space is flat only if the ratio $\Omega:=\rho / \rho_{c r} \cong 1$, where $\rho_{c r}:=3 H^{2} / 8 \pi G$ is the critical mass density (matter + radiation), $\rho_{c r}=10^{-29} \mathrm{~g} / \mathrm{sm}^{3}$. According to modern observational data, the value $\Omega=1.02 \pm 0.02$.
} 


$$
\frac{1}{a(t)} a_{t t} \equiv\left[\frac{d H(t)}{d t}+H(t)^{2}\right]=-\frac{4 \pi G}{3}\left(\rho(t)+\frac{3 P(t)}{c^{2}}\right)+\underset{\text { omitted }}{\Lambda / 3},
$$

which describe an expansion of the Universe. Here, the dot denotes the time derivatives; $H(t):=a_{t} / a$ - the Hubble parameter, or the Hubble expansion rate of the Universe (in the modern period $\left.H_{0}=2.2 \times 10^{-18} c^{-1}\right) ; \rho=\rho_{m}+\rho_{\gamma}$ - total density of matter and radiation. Equations (4) and (5) include an additional governing parameter $\Lambda / 3$ that, if properly defined, can explain the accelerated expansion of the late Universe [33].

From equations (4) and (5) it is easy to obtain the following continuity equation - "energy conservation law"

$$
\rho_{, t}(t)+3 \frac{a_{t}(t)}{a(t)}\left[\rho(t)+\frac{P(t)}{c^{2}}\right]=0 .
$$

To do this, it is necessary to differentiate (4) and combine the result with the ratio (5), which the pressure satisfies. Note that equation (6) can also be derived directly from the first law of thermodynamics, if we consider the Universe as a thermodynamic system bounded by the visible horizon and expanding adiabatically ([38], see also Section 5.1. of this work).

Equation (6) can be written as $a d \rho / d a=-3\left(\rho+P / c^{2}\right)$, or, which is the same

$$
d\left(\rho a^{3}\right) / d a=-3 P c^{-2} a^{2}
$$

If the dependence of pressure $P(t)$ on $a(t)$ is known, it is possible, by solving equation (4) (at $\Lambda=0$ ), to determine $a(t)$ for all times. Thus, the fundamental equations of dynamic cosmology are the Einstein equations (4), the energy conservation equation (6) and the equation of state.

Cosmological models based on the Robertson-Walker metric, in which $a(t)$ it is determined from these equations, are called Friedman models [39]. Note that the solution $a(t)$ obtained in this way automatically satisfies Eq. (5), since differentiating (4) with respect to time and using (7), we obtain

$$
2 a_{, t} a_{t t}=\frac{8 \pi G}{3 a} a_{t}\left[-\rho a^{2}+\frac{d}{d a}\left(\rho a^{3}\right)\right]=\frac{8 \pi G}{3 a} a_{t}\left(-\rho a^{2}-3 \frac{P}{c^{2}} a^{2}\right)
$$

which is equivalent to equation (5).

Equation (7) can be easily solved in the case of an equation of state in the form $P=w \rho$ with a time-independent coefficient $w$. In this case, equation (6) leads to a solution $P \sim a^{-3-3 w}$, which, in particular, is applicable in the following frequently encountered limiting cases:

- if the main contribution to the energy density of the Universe is made by nonrelativistic matter with negligible pressure, then it follows from (7) that

$$
\rho(t) \sim a(t)^{-3} \text { when } P<<\rho ;
$$


- if the contribution of relativistic particles, such as photons, prevails in the energy density, then $P=\rho / 3$, and from (7) one obtains

$$
\rho(t) \sim a(t)^{-4} \text { when } P=\rho / 3
$$

- in the case of a cosmological vacuum, when $P=-c^{2} \rho$, equation (7) has a solution in the form of a constant $\rho$, known (up to generally accepted numerical factors) as the cosmological constant $\Lambda$, or vacuum density.

The currently known observations of the accelerating expansion of the Universe are consistent with the existence of a constant vacuum energy equal to $\rho c^{2}$. The very existence of an accelerating expansion, in accordance with equation (5), requires that a significant part of the energy density of the Universe should be in such a form for which $\rho+3 P / c^{2}<0$, in contrast to ordinary matter and radiation. This form is called dark energy in cosmology [4].

\section{ACCELERATED EXPANSION OF THE UNIVERSE}

\subsection{Entropy force associated with the Bekenstein-Hawking entropy}

In this work, to explain the accelerated expansion of the Universe, we will use a different approach (without dark energy), in which the ideas of information, holography, entropy and temperature play a central role (see $[10,22,40]$ ). Consideration of the entropic force on the holographic horizon of an expanding flat Universe, which has associated entropy and temperature, leads to the so-called entropic cosmology, which assumes that it is the entropic force acting on the Hubble horizon and directed outward towards the horizon that is responsible for the phenomenon of accelerated expansion. For this reason, there is no ambiguous dark energy component in the cosmological equations.

With this approach, by analogy with the thermodynamic characteristics of the Hubble horizon of a black hole described by its temperature and entropy, entropy cosmology assumes that the region of the expanding flat Universe (coinciding with the Hubble horizon) has a temperature proportional to the de Sitter temperature [30] and the associated Bekenstein-Hawking entropy [15]. In this case, the problem of the relationship between the cosmological constant and the entropic force is solved in a natural way [10].

In entropic cosmology, the Hubble horizon (radius) $R_{H}$ and the temperature of the cosmological horizon of the Universe $T_{H} \simeq \gamma T_{S}$ are determined by the expressions [15]

$$
\begin{gathered}
R_{H}=c H^{-1}, \\
T_{H}=\gamma \frac{\hbar}{2 \pi k_{B}} H=\frac{\hbar}{2 \pi k_{B}} \frac{c}{R_{H}},
\end{gathered}
$$


where $k_{B}$ and $\hbar=h / 2 \pi$ are the Boltzmann constant and the reduced Dirac constant, respectively; $\gamma$ - non-negative free order parameter $O(1)$ (usually $\gamma \sim 1 / 2$ or $3 / 2 \pi$, which corresponds to the parameter for the screen temperature obtained in [15].

The temperature of the horizon of the Universe, closely related to the de Sitter temperature $T_{S}=\hbar H / 2 \pi k_{B}$, can be estimated as

$$
T_{H} \simeq \frac{\hbar H}{2 \pi k_{B}} \times \mathcal{O}(1) \sim 3 \times 10^{-30} K
$$

which is much lower than the temperature of the cosmic microwave background, $T=2.73 \mathrm{~K}$. The entropy associated with the horizon of the Universe is given by the following Bekenstein-Hawking relation [13]

$$
S_{B H}=k_{B}\left(\frac{A_{H}}{A_{P l}}\right)=k_{B} \frac{c^{3}}{\hbar G} A_{H}
$$

where $A_{H}$ is the size of the area of the standard horizon (surface area of the Hubble radius area $\left.R_{H}\right) ; \quad A_{P l}=\hbar G / c^{3} \approx 2.612 \times 10^{-70} m^{2}-$ Planck area. Substituting the quantity $A_{H}=\pi R_{H}^{2}=\pi c^{2} H^{-2}$ into relation (13), we obtain

$$
S_{B H}=k_{B}\left(\frac{c^{3}}{\hbar G}\right) \pi R_{H}^{2}=\left(\frac{k_{B} \pi c^{5}}{\hbar G}\right) \frac{1}{H^{2}} \equiv \frac{K}{H^{2}} \sim(2.6 \pm 0.3) \times 10^{122} k_{B} .
$$

A positive constant is introduced here

$$
K:=\frac{\pi k_{B} c^{5}}{\hbar G}=\frac{\pi k_{B} c^{2}}{L_{P l}^{2}}=\frac{\pi k_{B} c^{2}}{A_{P l}}>0
$$

where $L_{P l}=\sqrt{\hbar G / c^{3}}$ is the Planck length.

Increasing the radius $R_{H}$ by $d R_{H}$ increases the entropy $S_{B H}$ by $d S_{B H}$ in accordance with the formula

$$
\begin{gathered}
d S_{B H}=\left(\frac{k_{B} c^{3}}{\hbar G}\right) 2 \pi R_{H} d R_{H}= \\
=\left(\frac{k_{B} c^{3}}{\hbar G}\right) 2 \pi\left(\frac{c}{H}\right) d R_{H} \sim(2.6 \pm 0.3) \times 10^{122} k_{B} \frac{d R_{H}}{R_{H}} .
\end{gathered}
$$

The entropy force $F_{B H}$ corresponding to the growth of the Bekenstein-Hawking entropy can be defined as 


$$
F_{B H}=-T_{H} \frac{d S_{B H}}{d r_{H}}
$$

Here, the minus sign indicates the direction of increasing entropy or screen, which in this case is the event horizon [15].

Substituting now relations (11) and (13) into (16) and using the formula for the area $A_{H}$ of the standard horizon, we obtain the following expression for the entropic force

$$
\begin{aligned}
F_{B H} & =-T_{H} \frac{d S_{B H}}{d R_{H}}=-\gamma \frac{\hbar H}{2 \pi k_{B}} \times \frac{d}{d r_{H}}\left[\frac{K}{H^{2}}\right]=\frac{2 \hbar H}{2 \pi k_{B}} \frac{K}{H^{3}} \frac{d H}{d r_{H}}= \\
& =\gamma \frac{1}{H^{2}} \frac{c^{5}}{G} \frac{d H}{d R_{H}}=-\gamma \frac{1}{H^{2}} \frac{c^{6}}{G} \frac{1}{R_{H}^{2}}=-\gamma \frac{c^{4}}{G} .
\end{aligned}
$$

The pressure $P_{B H}$ of this force on the cosmological horizon of the Universe is determined by the formula

$$
P_{B H}=\frac{F_{B H}}{4 A_{H}}=-\gamma \frac{c^{4}}{G} \frac{1}{4 \pi R_{H}^{2}}=-\gamma \frac{c^{2}}{4 \pi G} H^{2}=-\gamma \frac{2 \rho_{c r}}{3} c^{2}
$$

(where $\rho_{c r}:=3 H^{2} / 8 \pi G$ is the critical mass density of matter and radiation). This value is close to the measured negative pressure (tension) of dark energy in the form of a cosmological constant [4]. Thus, in the holographic approach, pressure arises not due to the negative pressure of dark energy, but due to the entropic tension due to the entropic content on the horizon of the Universe. The presence of such tension is equivalent to outward cosmic acceleration ${ }^{\text {viii) }}$. In other words, the acceleration of the universe arises as a natural consequence of the entropy change at the horizon of the Universe.

\subsection{Accelerated expansion of the Universe under the influence of the Bekenstein-Hawking entropy force}

We will now assume that in entropy cosmology the effective pressure $P_{B H}^{\prime}$ based on the Bekenstein-Hawking entropy is determined by the relation

$$
P_{B H}^{\prime}=P+P_{B H}=P-\gamma \frac{c^{2}}{4 \pi G} H^{2} .
$$

When using $P_{B H}^{\prime}$, equations (5) and (6) take the following form:

viii) Note that from the possibility of describing the cosmic acceleration of the Universe by an entropic force, it does not follow that gravity itself is an entropic force [10]. 


$$
\begin{aligned}
& \frac{a_{\prime t t}}{a}=-\frac{4 \pi G}{3}\left(\rho(t)+\frac{3 P(t)}{c^{2}}\right)+\gamma H(t)^{2}, \\
& \rho_{t_{t}}+3 H(t)\left[\rho(t)+\frac{P}{c^{2}}\right]=\gamma \frac{3}{4 \pi G} H(t)^{3} .
\end{aligned}
$$

These equations can be considered as modified equations of acceleration (5) and continuity (6) for entropy cosmology, obtained using the Bekenstein-Hawking entropy. The quantity $H^{2}$ in these equations is related to the entropic force, which can explain the accelerated expansion of the Universe without introducing the concept of dark energy - the cosmic vacuum (associated with the cosmological constant), the energy density of which is negative. Note that the Beken-stein -Hawking entropy is proportional to the area of the cosmological horizon of the Universe, due to which the model based on this entropy predicts only the Universe expanding with uniform acceleration. This model of the accelerated expansion of the Universe is capable to provide a good fit with supernova data $[15,22]$.

\section{ENTROPIC FORCE ASSOCIATED WITH NON-ADDITIVE ENTROPY OF BARROW AND TSALLIS-CIRTO}

Recently [35] proposed a model of the quantum gravitational foam of space-time was proposed to estimate the entropy of black holes and the Universe, the surface of which can have a complex fractal structure of the cosmological horizon down to arbitrarily small scales (up to a scale of the order of the Planck length) due to quantum gravitational effects. The introduction of the fractal structure of the horizon (space-time region) of the Universe leads to an increase in its surface area. As you know, the surface area of the Universe is a key characteristic that determines its entropy and information content.

The complex fractal structure of the horizon of the Universe results in a finite volume, but with an infinite (or finite) area [35]. According to the thermodynamics of black holes, the possible effects of the quantum-gravitational foam of space-time in the region of the cosmological horizon lead to a new definition of the entropy of the Universe - to the non-additive entropy of Barrow $S_{B}$ [35] related to the additive Bekenstein-Hawking entropy as follows: $S_{B} / k_{B}=\left(S_{B H} / k_{B}\right)^{1+D / 2}$. Substituting the values $S_{B H}$ and $k_{B}$ into this ratio yields $S_{B} \sim 10^{120(1+D / 2)}$. Here, the parameter $D(0 \leq D \leq 1)$ is the fractal mass dimension of the quantum-gravitational foam, which quantitatively determines the deformation of the structure of the horizon of the Universe ${ }^{\mathrm{ix})}$.

ix) It should be noted that when defining the Barrow entropy, the complex fractal structure of the cosmological horizon is modeled by an analogue of the spherical "Koch snowflake" using an infinite decreasing hierarchy of touching spheres around the Schwarzschild event horizon. Nevertheless, this simple model of possible manifestations of quantum-gravitational effects has important implications for estimates of the entropy of the Universe, which is usually slightly larger than in the baseline scenario. 
It is easy to show that Barrow's entropy obeys the following pseudo-additive law for two independent systems $\mathrm{N}$ and $\mathrm{M}$ :

$$
\frac{S_{B}(\mathrm{~N}+\mathrm{M})}{k_{B}}=\left(\left[\frac{S_{B}(\mathrm{~N})}{k_{B}}\right]^{\frac{2}{2+D}}+\left[\frac{S_{B}(\mathrm{M})}{k_{B}}\right]^{\frac{2}{2+D}}\right)^{\frac{2+D}{2}} .
$$

Entropy $S_{B}$ can be written as follows:

$$
\begin{gathered}
S_{B}=k_{B}\left(\frac{A_{H}}{A_{P l}}\right)^{1+D / 2}=k_{B}\left(\frac{\pi R_{H}^{2}}{A_{P l}}\right)^{1+D / 2}=\frac{k_{B} \pi c^{3}}{\hbar G} R_{H}^{2}\left(\frac{\pi R_{H}^{2}}{A_{P l}}\right)^{D / 2}= \\
=K c^{-2} R_{H}^{2}\left(\frac{\pi R_{H}^{2}}{A_{P l}}\right)^{D / 2}=K c^{-2} R_{H}^{2}\left(\frac{K}{k_{B}} R_{H}^{2}\right)^{D / 2}=\left(\frac{K^{1+D / 2}}{c^{2} k_{B}^{D / 2}}\right) R_{H}^{2+D} .
\end{gathered}
$$

Here $A_{P l}=\hbar G / c^{3} \approx 2.612 \times 10^{-70} m^{2}$ - Planck area; $K:=\pi k_{B} c^{2} / A_{P l}>0 ; A_{H}$ - standard horizon area; In the case $D=0$ that corresponds to the simplest structure of the cosmological horizon of the Universe, the standard $S_{B} \equiv S_{B H}=k_{B}\left(A_{H} / A_{P l}\right)$ Bekenstein-Hawking entropy considered above is restored.

When $D=1$, then there is a smooth space-time structure of the horizon of the Universe, at which $S_{B} \equiv S_{T C}=k_{B}\left(\frac{A_{H}}{A_{P l}}\right)^{3 / 2}$. In this case, formula (22) is similar to that for the non-additive entropy of Tsallis and Cirto [31], introduced by these authors, when studying the evolution of a black hole on the basis of completely different physical principles, different from the fractal interpretation (see [41-44]). In order to obtain modified cosmological equations, we apply the procedure considered in the previous section to derive an expression for the entropy force, but now involving the Barrow entropy (22). It is evident that in the general case of a medium with fractal dimension $(0<D \leq 1)$, these equations, in contrast to the Friedman equations (20) and (21), will contain new additional terms that allow modeling the cosmological behavior of the Universe [34, $36,45]$.

\subsection{Entropic Force Associated with Barrow's Entropy}

At this point, we will consider the possibility of an accelerated cosmological expansion of the Universe, but using the Barrow entropy at its horizon. Barrow's entropy arises, in particular, due to the fact that the surface of the horizon of the Universe can deform due to quantumgravitational effects, and its deviation from the Bekenstein-Hawking entropy is quantitatively determined by the fractal dimension index $D$.

Increasing the radius $R_{H}$ by $d R_{H}$ increases the entropy $S_{B}$ by $d S_{B}$ in accordance with the expression 


$$
d S_{B}(D)=k_{B}(2+D)\left(\pi / A_{P l}\right)^{1+D / 2} R_{H}^{1+D} d R_{H}
$$

Then for the entropic force $F_{B}$ arising from the modification of the horizon of the Universe, which is associated with quantum-gravitational effects, we will have:

$$
\begin{aligned}
F_{B} & =-T_{H} \frac{d S_{B}}{d R_{H}}=-\gamma\left(\frac{\hbar c}{2 \pi k_{B} R_{H}}\right) k_{B} \frac{\pi c^{3}}{\hbar G}\left(\frac{\pi}{A_{P l}}\right)^{D / 2}(2+D) R_{H}^{1+D}= \\
& =-\gamma \frac{2+D}{2} \frac{c^{4}}{G}\left(\frac{\pi}{A_{P l}}\right)^{D / 2} R_{H}^{D}=-\gamma \frac{(2+D)}{2} \frac{c^{4+D}}{G}\left(\frac{K}{k_{B}}\right)^{D / 2} H^{-D} .
\end{aligned}
$$

Accordingly, the pressure $P_{B}$ of this force on the cosmological horizon of the Universe is defined by the formula

$$
P_{B}=\frac{F_{B}}{4 A_{H}}=-\gamma \frac{c^{4}}{4 \pi G}\left(\frac{K}{k_{B}}\right)^{D / 2} \frac{(2+D)}{2} R_{H}^{D-2}=\gamma \frac{(2+D)}{2} \frac{c^{2+D}}{4 \pi G}\left(\frac{K}{k_{B}}\right)^{D / 2} H^{2-D} .
$$

In what follows, we will assume that in entropy cosmology the effective pressure $P_{B}^{\prime}$ based on the Barrow entropy is determined by the relation

$$
P_{B}^{\prime}=P+P_{B}=P-\gamma \frac{(2+D)}{2} \frac{c^{2+D}}{4 \pi G}\left(\frac{K}{k_{B}}\right)^{D / 2} H^{2-D}
$$

When using $P^{\prime}{ }_{B}$ the equations of acceleration (5) and continuity (6) take the form

$$
\begin{gathered}
\frac{1}{a(t)} a_{, t t}=-\frac{4 \pi G}{3}\left(\rho(t)+\frac{3}{c^{2}} P(t)\right)+\gamma \frac{(2+D)}{2} c^{D}\left(\frac{K}{k_{B}}\right)^{D / 2} H(t)^{2-D}, \\
\rho_{, t}(t)+3 H(t)\left[\rho(t)+\frac{P(t)}{c^{2}}\right]=\gamma \frac{3}{4 \pi G} \frac{2+D}{2} c^{D}\left(\frac{K}{k_{B}}\right)^{D / 2} H(t)^{3-D} .
\end{gathered}
$$

It is important to note that in the case of fractal dimension $D=0$ these equations will coincide with the modified Friedman equations (20) and (21), i.e., the deformation of the Bekenstein- Hawking holographic entropy is measured by a new index $D$, whereas the case of zero defor-mation ( $D=0$ ) corresponds to the entropy force Barrow, which fully complies with the stand-ard entropy force considered in [15].

At the same time, the authors of [34], based on observational data from a sample of the collec-tion (SNIa) of supernovae and using direct measurements of the Hubble parameter by cosmic chronometers, showed that the value deformation parameter equal to $D=0.094$, assuming that a small deviation from the standard holographic Bekenstein-Hawking entropy is preferable. 
The case $D=1$ corresponds to the maximum deformation associated with the Tsallis-Cirto cosmological entropy [31]. The scenario for the manifestation of this entropy predicts both deceleration and/or accelerated expansion of the Universe [46].

In the general case, when $0<D<1$ we have a new cosmological scenario for the manifestation of the entropic force, based on the Barrow entropy associated with the quantumgravitational effects of the horizon of the Universe. This scenario allows simulating the cosmological behavior of the Universe for the case of various modifications of Barrow's governing gravitational force [36].

\subsection{Entropic force associated with the entropy of Tsallis-Cirto}

Let us now consider entropic cosmology under the assumption that the cosmological horizon of the Universe has a temperature

$$
T_{H}=\gamma \frac{\hbar}{2 \pi k_{B}} \frac{c}{R_{H}}=\gamma \frac{\hbar}{2 \pi k_{B}} H
$$

and that the non-additive entropy of Tsallis-Cirto, is defined as follows [31]

$$
\begin{aligned}
S_{T C} & =S_{B}(1):=k_{B}\left(\frac{A_{H}}{A_{P l}}\right)^{3 / 2}=k_{B}\left(\frac{\pi R_{H}^{2}}{A_{P l}}\right)^{3 / 2}=\frac{k_{B} \pi c^{3}}{\hbar G} R_{H}^{2}\left(\frac{\pi R_{H}^{2}}{A_{P l}}\right)^{1 / 2}= \\
& =K c^{-2}\left(\frac{\pi}{A_{P l}}\right)^{1 / 2} R_{H}^{3}=\left(\frac{K^{3}}{k_{B} c^{4}}\right)^{1 / 2} R_{H}^{3}=c K\left(\frac{K}{k_{B}}\right)^{1 / 2} \frac{1}{H^{3}} .
\end{aligned}
$$

It follows from formula (29) that the non-additive entropy $S_{T C}$ is proportional to the volume of the horizon of the Universe, in contrast to the Bekenstein-Hawking entropy (14), which is proportional to its area.

Increasing the radius $R_{H}$ by $d R_{H}$ increases the Tsallis-Cirto entropy $d S_{T C}$ in accordance with the ratio

$$
d S_{T C}=3\left(K^{3} / k_{B} c^{4}\right)^{1 / 2} R_{H}^{2} d R_{H}
$$

Using (30), we obtain the following expressions for the entropy force and pressure on the cosmic horizon of the Universe, corresponding to the Tsallis-Cirto entropy:

$$
\begin{gathered}
F_{T C}=-T_{H} \frac{d S_{B}}{d R_{H}}=-\gamma \frac{3}{2} \frac{c^{4}}{G}\left(\frac{\pi}{A_{P l}}\right)^{1 / 2} R_{H}= \\
=-\gamma \frac{3}{2}\left(\frac{\hbar}{\pi k_{B} c}\right)\left(\frac{K^{3}}{k_{B}}\right)^{1 / 2} R_{H}=-\gamma \frac{3}{2}\left(\frac{c^{4}}{K G}\right)\left(\frac{K^{3}}{k_{B}}\right)^{1 / 2} R_{H},
\end{gathered}
$$




$$
P_{T C}=\frac{F_{T C}}{4 \pi R_{H}^{2}}=-\frac{3}{2} \gamma \frac{c^{4}}{4 \pi G}\left(\frac{K}{k_{B}}\right)^{1 / 2} R_{H}^{-1}=-\gamma \frac{c^{2}}{4 \pi G} \frac{3 c}{2}\left(\frac{K}{k_{B}}\right)^{1 / 2} H
$$

Assuming, as it was made before, that in entropy cosmology the effective pressure $P_{T C}^{\prime}$ based on the Tsallis-Cirto entropy is determined by the relation $P_{T C}^{\prime}=P+P_{T C}$, and substituting $P_{T C}^{\prime}$ in the equations of acceleration (5) and continuity (6); we will obtain:

$$
\begin{gathered}
\frac{a_{t t}}{a}=-\frac{4 \pi G}{3}\left(\rho(t)+\frac{3 P(t)}{c^{2}}\right)+\frac{3 c}{2}\left(\frac{K}{k_{B}}\right)^{1 / 2} \gamma H(t), \\
\rho_{t_{t}}+3 H(t)\left[\rho(t)+\frac{P(t)}{c^{2}}\right]=\frac{3}{4 \pi G} \frac{3 c}{2}\left(\frac{K}{k_{B}}\right)^{1 / 2} \gamma H(t)^{2} .
\end{gathered}
$$

Equations (33) and (34) can be considered as modified equations of acceleration and continuity based on the generalized Tsallis-Cirto entropy. From equation (33) it follows that the governing force term in this model is proportional to the Hubble rate $H$ expansion of the Universe, in contrast to the analogous entropy force term in the Bekenstein-Hawking model, which is proportional to $H^{2}$.

It should be noted that cosmological equations similar to equations (33) and (34) have been repeatedly discussed in the literature when modeling the evolution of the Universe based on different approximations of the variable cosmological term (see, for example, [46]). On the other hand, the entropy force (31) obtained from the generalized entropy Tsallis-Cirto behaves in the same way as the driving force of a viscous cosmological fluid with bulk viscosity $\eta$, which is used to explain the accelerated expansion of the Universe in models of viscous cosmology. Indeed, the expression for the effective pressure $P_{T C}^{\prime}(t)=P(t)-\frac{3 c^{3}}{8 \pi G}\left(\frac{K}{k_{B}}\right)^{1 / 2} H(t)$ in equation (34) is similar to the expression $P^{\prime}(t)=P(t)-3 \eta H(t)$ for pressure in viscous cosmology models designed to simulate dark matter. Models of this type assume that the Universe is filled with a cosmological fluid with bulk viscosity that can generate the entropy of a homogeneous and isotropic Universe (see [47-51). This similarity became possible due to the fact that the nonadditive entropy of Tsallis Cirto, introduced on the basis of the holographic principle, behaves as if it were the classical entropy of a homogeneous and isotropic Universe generated by the volumetric viscous stress of a cosmological fluid [48, 52-54].

Thus, using the holographic principle, which is associated with the existence of the Barrow entropy on the horizon of the Universe, in this work two models of the entropic force were considered: model (17) based on the Bekenstein-Hawking entropy, and model (31) based on nonadditive Tsallis-Cirto entropy. These models describe the evolution of an accelerating Universe without using the concept of the cosmological constant or dark energy. 
This implies that the Bekenstein-Hawking entropy force model predicts a uniformly accelerating Universe, while the Tsallis-Cirto model predicts both deceleration and accelerated expansion of the Universe $[46,55]$.

\section{THERMODYNAMIC APPROACH TO THE DEVELOPMENT OF THE EQUATION OF ENERGY CONSERVATION}

Let us now proceed to consideration of the non-adiabatic expansion of the Universe caused by Barrow's cosmological entropy on the Hubble horizon. For this purpose, we derive the generalized energy equation (6), modifying the thermodynamic approach developed in the monograph [38].

\subsection{Adiabatic expansion of the Universe}

According to the first law of thermodynamics, the principle of conservation of total energy for non-additive systems can be written in the form $d Q / d t=d E / d t+P d V / d t$ or in the form of the Gibbs relation $[56,57]$

$$
T d S / d t=d Q / d t=d E / d t+P d V / d t
$$

expressing the rate TdS / Dt of change in entropy $S$ when an element of a non-additive medium moves along its trajectory. Here $d Q$ is the heat transferred across the border from the environment to the element of the environment, $d E$ and $d V$ changes in the internal energy and volume of the area of matter and radiation, respectively. Relation (35) can be rewritten as

$$
T d S=d Q=T \frac{d S}{d t} d t=\frac{d E+P d V}{d t} d t=\left(E_{,_{t}}+P V_{, t}\right) d t
$$

Let us now consider a sphere of initial radius $\hat{r}_{S}$, expanding together with the universal expansion of the Universe, so that its own radius $R_{H}(t)$ at the moment of time $t$ is determined by the expression $R_{H}=a(t) \hat{r}_{s}$. Then the volume $V(t)$ of the sphere is

$$
V(t)=(4 \pi / 3) \hat{r}_{s}^{3} a(t)^{3}
$$

From this it follows

$$
V_{t_{t}}=\frac{4 \pi}{3} \hat{r}_{s}^{3}\left(3 a^{2} a_{t}\right)=V\left(3 \frac{a_{t}}{a}\right)=3 V H, \quad\left(a_{t} / a=H\right) .
$$

For the internal energy of the sphere, we have $E(t)=\varepsilon(t) V(t)$, where $\varepsilon(t)$ is the internal energy density, determined by the relation $\varepsilon(t)=\rho(t) c^{2}$. Hence, the rate of change in the internal energy of the sphere $E(t)$ is determined as

$$
E_{r_{t}}=\varepsilon_{r_{t}} V+\varepsilon V_{t_{t}}=\left(\varepsilon_{r_{t}}+3 H \varepsilon\right) V
$$


Substituting equations (38) and (39) into $E_{t_{t}}+P V_{,_{t}}$, we obtain

$$
\begin{aligned}
E_{t_{t}}+P V_{t_{t}} & =\left(\varepsilon_{r_{t}}+3 H \varepsilon\right) V+3 P V H=\left[\varepsilon_{t_{t}}+3 H(\varepsilon+P)\right] V= \\
& =\left[\rho_{t_{t}}+3 H\left(\rho+P / c^{2}\right)\right] c^{2} V .
\end{aligned}
$$

Finally, substituting relations (37) and (39) into equation (36), we obtain the first law of thermodynamics for an expanding or contracting Universe:

$$
\begin{aligned}
T \frac{d S}{d t} & =\left(E_{t_{t}}+P V_{t}\right)=\left[\rho_{t}+3 H\left(\rho+\frac{P}{c^{2}}\right)\right] c^{2} V= \\
& =c^{2}\left[\rho_{t_{t}}+3 H\left(\rho+P / c^{2}\right)\right](4 / 3) \pi R_{H}^{3} .
\end{aligned}
$$

Let us now consider such motions of cosmic matter for which the entropy of each particle of the medium remains in the first approximation constant throughout the entire path of an element of the medium, i.e. $d S / d t=0$. Such reversible and adiabatic motions are isentropic. For them, equation (41) is reduced to the previously obtained continuity equation (6) for the adiabatic expansion of the Universe

$$
\rho_{t_{t}}(t)+3 \frac{a_{t}(t)}{a(t)}\left[\rho(t)+P(t) / c^{2}\right]=0 .
$$

\subsection{Modified energy equation for modeling non-adiabatic expansion of the Universe}

If the evolution of the Universe within the framework of non-adiabatic entropic cosmology is modeled, then $d S / d t \neq 0$ (see [58-60]. To calculate $T d S / d t$ in equation (41), we will use formula (24) related to the Barrow entropy [35], as the most general in the case under consideration. As a result, we will have

$$
\begin{gathered}
T_{H} \frac{d S_{B}}{d t}=\gamma\left(\frac{\hbar c}{2 \pi k_{B} R_{H}}\right) k_{B} \frac{\pi c^{3}}{\hbar G}\left(\frac{\pi}{A_{P l}}\right)^{D / 2}(2+D) R_{H}^{1+D} \frac{d R_{H}}{d t}= \\
=\gamma \frac{2+D}{2} \frac{c^{4}}{G}\left(\frac{\pi}{A_{P l}}\right)^{D / 2} R_{H}^{D} \frac{d R_{H}}{d t}=-\gamma \frac{(2+D)}{2} \frac{c^{5+D}}{G}\left(\frac{K}{k_{B}}\right)^{D / 2} H^{-D-2} H_{, t} .
\end{gathered}
$$

Taking into account expression (42), the energy equation (41)

$$
\left[\rho_{, t}+3 H\left(\rho+\frac{P}{c^{2}}\right)\right]=T_{H} \frac{d S_{B}}{d t} c^{-2}\left(\frac{3}{4 \pi} R_{H}^{-3}\right)
$$


in the case of non-adiabatic expansion of the Universe under the influence of the driving entropy force (associated with the Barrow entropy) takes the form

$$
\rho_{, t}+3 \frac{a_{t}}{a}\left(\rho+\frac{P}{c^{2}}\right)=-\frac{D+2}{2} c^{2 D}\left(\frac{K}{k_{B}}\right)^{D / 2}\left(\frac{3}{4 \pi G}\right) \gamma H^{1-D} H_{,_{t}} .
$$

This is a modified equation of continuity obtained from the first law of thermodynamics under the assumption of non-adiabatic expansion of the Universe. The right-hand side of equation (43) is associated with a non-adiabatic process. If $H=0$ or if $H=$ const, then equation (43) is reduced to the continuity equation for the adiabatic expansion of the Universe. Note that a similar modification of the continuity equation for entropy cosmology has been studied for other cosmological models of the expansion of the Universe (see, for example, [18, 19].

Using equation (43), the following equations of continuity can be obtained in the case of nonadiabatic expansion of the Universe under the influence of the Bekestein-Hawking and Tsallis-Cirto entropic forces:

$$
\begin{gathered}
\rho_{t_{t}}+3 \frac{a_{t}}{a}\left(\rho+\frac{P}{c^{2}}\right)=-\gamma\left(\frac{3}{4 \pi G}\right) H H_{t_{t}}, \quad(D=0), \\
\rho_{t_{t}}+3 \frac{a_{t}}{a}\left(\rho+\frac{P}{c^{2}}\right)=-\gamma \frac{3 c^{2}}{2}\left(\frac{K}{k_{B}}\right)^{1 / 2}\left(\frac{3}{4 \pi G}\right) H_{, t}, \quad(D=1) .
\end{gathered}
$$

\subsection{Simple models of non-adiabatic expansion of the Universe}

In this subsection, using the modified continuity equation (43), we'll analyze two generalized Friedman equations (4) and (5) for the scale factor in the case of non-adiabatic expansion of the Universe under the influence of the Barrow entropy force.

For this purpose, we write equation (4) in the form

$$
(a, t)^{2}=\frac{8 \pi G}{3} \rho a^{2}+f(t) a^{2}
$$

where $f(t)$ is a function depending on the type of entropy force, including high-order corrections. Differentiating this equation with respect to $t$, we obtain

$$
2 a_{,_{t}} a_{t_{t t}}=\frac{8 \pi G}{3}\left(\rho_{t_{t}} a^{2}+2 \rho a a_{t}\right)+f_{t_{t}} a^{2}+2 f a a_{, t},
$$

or, after dividing by $2 a a_{t}$,

$$
\frac{a_{\prime t t}}{a}=\frac{4 \pi G}{3}\left(\frac{1}{H} \rho_{\prime_{t}}+2 \rho\right)+\frac{1}{2 H} f_{,_{t}}+f .
$$

Now multiplying the energy equation (43) by $a / a_{t}=1 / H$, as a result we will have 


$$
\frac{\rho_{t}}{H}=-3(1+w) \rho-\frac{D+2}{2} c^{2 D}\left(\frac{K}{k_{B}}\right)^{D / 2}\left(\frac{3}{4 \pi G}\right) \gamma H^{-D_{H}} H .
$$

where the notation is introduced $w=P / \rho c^{2}$. Substituting relation (48) into equation (47), we finally obtain

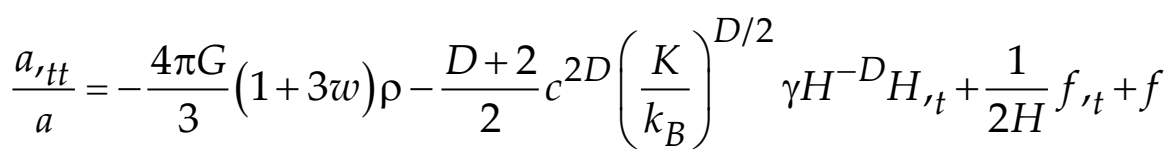

Further, for the purpose of simulation, one should follow the work [15, 22] concept, where it was assumed that the term associated with the entropy force does not depend on the time derivative of the Hubble parameter. Following this assumption, we define the functions $f(t)$ in such a way that the term with $H_{, t}$ is absent in equation (49). If we put

$$
f(t)=\gamma \frac{2+D}{2-D} c^{2 D}\left(\frac{K}{k_{B}}\right)^{D / 2} H(t)^{2-D},
$$

we then obtain the following simple system of self-consistent equations, composed of the modified Friedman equations, acceleration and continuity:

$$
\begin{gathered}
\left(\frac{a_{t}}{a}\right)^{2}=\frac{8 \pi G}{3} \rho a^{2}+\gamma \frac{2+D}{2-D} c^{2 D}\left(\frac{K}{k_{B}}\right)^{D / 2} H^{2-D}, \\
\frac{a_{t t}}{a}=-\frac{4 \pi G}{3}(1+3 w) \rho+\gamma \frac{2+D}{2-D} c^{2 D}\left(\frac{K}{k_{B}}\right)^{D / 2} H^{2-D}, \\
\rho_{t}+3 \frac{a_{t}}{a}\left(\rho+\frac{P}{c^{2}}\right)=-\frac{D+2}{2} c^{2 D}\left(\frac{K}{k_{B}}\right)^{D / 2}\left(\frac{3}{4 \pi G}\right) \gamma H^{1-D} H_{t_{t}} .
\end{gathered}
$$

This system of equations makes it possible to simulate a new scenario of the evolution of the Universe, if one considers it as a thermodynamic system bounded by the visible horizon, which expands nonadiabatically under the influence of the entropic force associated with the nonadditive entropy of Barrow.

Using the system of equations (51)-(53), it is possible to obtain a number of models that describe the non-adiabatic evolution of the Universe without using the concept of the cosmological constant, or dark energy. These models include, in particular, the non-adiabatic model based on the Bekenstein-Hawking entropy and the non-adiabatic model based on the Tsallis-Cirto nonadditive entropy. 
Assuming parameter $D=0$ in formula (50), we will have $f(t)=\gamma H(t)^{2}$ for the function $f(t)$. In this case, a simple entropy model of the non-adiabatic expansion of the Universe based on the Bekenstein-Hawking entropy takes the form

$$
\begin{gathered}
\left(a_{t}\right)^{2}=\frac{8 \pi G}{3} \rho a^{2}+\gamma H^{2}, \\
\frac{a_{t t}}{a}=-\frac{4 \pi G}{3}(1+3 w) \rho+\gamma H^{2}, \\
\rho_{t_{t}}+3 \frac{a_{t}}{a}\left(\rho+\frac{P}{c^{2}}\right)=-\gamma\left(\frac{3}{4 \pi G}\right) H H_{t_{t}} .
\end{gathered}
$$

Entropic force $f=\gamma H^{2}$ in the equation. (54) coincides with the corresponding term in formula (55). The modified Friedman equations (54) and (55) correspond to equations (4) and (5) of the Friedman cosmological model. Using this system, it is possible to establish a number of properties of a non-adiabatically expanding Universe (see, for example, [23, 25].

If we put $D=1$ in formula (50), then for the function $f(t)$ we obtain the following expression

$$
f=3 \gamma c^{2}\left(K / k_{B}\right)^{1 / 2} H
$$

and taking it into account the system (51)-(53) takes the form

$$
\begin{gathered}
\left(\frac{a_{t}}{a}\right)^{2}=\frac{8 \pi G}{3} \rho a^{2}+\gamma 3 c^{2}\left(\frac{K}{k_{B}}\right)^{1 / 2} H, \\
\frac{a_{t t}}{a}=-\frac{4 \pi G}{3}(1+3 w) \rho+\gamma 3 c^{2}\left(\frac{K}{k_{B}}\right)^{1 / 2} H, \\
\rho_{t}+3 \frac{a_{t}}{a}\left(\rho+\frac{P}{c^{2}}\right)=-\gamma \frac{3 c^{2}}{2}\left(\frac{K}{k_{B}}\right)^{1 / 2}\left(\frac{3}{4 \pi G}\right) H_{t} .
\end{gathered}
$$

This system of equations underlies the simulation of the evolution of the non-adiabatically expanding Universe under the influence of the Tsallis-Cirto entropy force [31].

Thus, entropy cosmology using the procedure of "gravitational thermodynamics" using the Barrow entropy is quite effective for constructing a number of models describing the evolution of the Universe and allowing one to find quantitative estimates of the non-adiabatic accelerated expansion of the Universe in accordance with observational data. 


\section{CONCLUSION}

Progress in astrophysics rooted in the ground-based and space astronomy greatly influenced the key concepts of our views about space environment, origin, evolution and fate of our Universe. For less than half a century since the beginning of space exploration [61] cosmology experienced dramatic changes and this process continuously escalates. New projects and breakthroughs in theoretical approaches in the coming years open extremely challenging horizons in this intriguing branch of astrophysics and general science.

The modern cosmological data indicate that the Universe is expanding with acceleration. Unfortunately, a simple modified general relativity, which includes a key parameter - the cosmological constant $\Lambda$, characterizing an expansion cannot describe this phenomenon convincingly enough. Therefore, it becomes necessary to search for an approach that could be used to describe the accelerated expansion of the Universe more effectively.

One of the directions along this path consists in the construction of a modified theory of gravity, according to which the entropic force underlies the accelerated expansion of the Universe. The emergence of this force is an inevitable consequence of the growth of entropy at the post-inflationary stage of the quantum canvas of space-time, which can be associated with the storage of holographic information on the "surface screen of the Universe", similar in a certain sense to the event horizon of a black hole.

It should be noted that the holographic principle was put forward earlier in the study of physics of the black holes as an important property of quantum gravity, which states that the properties of space are encoded at its boundary (on the gravitational horizon of events). Based on this principle, Verlinde proposed an extended holographic picture in which Einstein's gravity arises from the statistical effect of a holographic screen. This approach proved to be effective for describing quantitatively the accelerated expansion of the Universe. A number of authors generalized the basic scenario of the evolution of the Universe, based on the use of entropic forces of various nature, with involvement of the assumption that the horizon of the Universe, like the event horizon of a black hole, has its own entropy and temperature.

Recently, Barrow proposed a model of the quantum gravitational canvas of space-time to estimate the entropy of black holes and the Universe, the surface of which can have a complex fractal structure of the cosmological horizon down to arbitrarily small scales (an order of the Planck length) due to quantum gravitational effects. As it is known, many solutions of the classical Einstein equations, in particular, the isotropic homogeneous cosmological model of Friedman-Robertson-Walker, contain singularities and cannot be analytically continued beyond them. In this regard, we face the fundamental problem of modern cosmology: what caused the growth of fluctuations and the emergence of a fragment of space-time on the infinite quantum canvas of the Universe, which concentrated within itself a huge energy ("vacuum energy") and why and how it was followed by inflation (de Sitter phase) and subsequently, the Big Bang left behind observed echo in the form of microwave background radiation (CMB). One way or another, the basis of such a scenario, which gave rise to the birth of the Universe, is addressed to quantum-gravitational effects [62].

In this work, an attempt is undertaken to better understand the physical mechanism of the accelerated expansion of a flat, homogeneous and isotropic Universe. Modified cosmological equations are obtained, containing new additional terms that coincide with the basic Friedman equations in the case when the Barrow deformation exponent corresponds to the fractal dimension $D=0$. However, in the general case $0<D \leq 1$, new governing terms appear associated with 
changes in the entropy of Barrow on the Hubble horizon of the Universe. It significantly exceeds its age, which affect the evolution of the main cosmological criteria, such as the scale factor, the deceleration parameter, the density of matter (involving visible and dark matter, radiation, neutrinos, etc.) and the growth of linear perturbations of matter. This should lead to a new phenomenological description of the thermal history of the Universe. The core for this conclusion is based on the results of this work, in which the validity of using the generalized second law of thermodynamics for the Barrow entropy was thoroughly investigated. On this basis, modified Friedman equations were obtained, which made it possible to explain the non-adiabatic expansion of the Universe in terms of entropy, without involving hypothetical dark energy as a texture (fabric) of the very expanding space. It seems reasonable to find out in this approximation a solution to the modified Einstein equations with quantum corrections and to establish whether there are physically interesting nonsingular solutions among them.

As one may see, entropy cosmology, based on the concepts of "gravitational thermodynamics" using the entropy of Barrow, is regarded as quite effective approach for quantitative assessment of the non-adiabatic accelerated expansion of the Universe and its possible change with time. The results of the analysis of possible solutions of the cosmological equations analyzed in this work will be presented in the following publications of the authors.

\section{REFERENCES}

[1] J.A.S. Lima, J. S. Alcaniz, "Constraining the cosmic equation of state from old galaxies at high redshift", Mon. Not. R. Astron. Soc. 317, 893-896 (2000).

[2] E. Komatsu, et al. "Seven-year Wilkinson Microwave Anisotropy Probe (WMAP) Observations: Cosmological Interpretation”, Astrophys. J. Suppl. Ser. 192(2). article id. 18, 47 pp. (2011).

[3] Ch.W. Mizner, K.S. Torn, J.A. Wheeler, Gravitatsiya. W.H. Freeman and Company. San Francisco. (1973).

[4] S. Weinberg, Cosmology. Oxford University Press. (2008).

[5] D.S. Gorbunov, V.A. Rubakov, "Introduction to the theory of the early universe: hot big bang theory", British Library Cataloguing-in-Publication Data. (2018).

[6] E.J. Copeland, M. Sami, S. Tsujikawa, "Dynamics of dark energy", Int. J. Mod. Phys. D. 15(11), 1753- 1935 (2006).

[7] Y.-F. Cai, E.N. Saridakis, M.R. Setare, J-Q. Xia, "Quintom Cosmology: Theoretical implications and observations", Phys. Rept. 493,1-60 (2010).

[8] A.M. Cherepashchuk, A.D. Chernin, Vselennaya, zhizn', chernyye dyry. Fryazino: «Vek 2». (2004).

[9] M.Ya. Marov, Kosmos: Ot Solnechnoy sistemy vglub' Vselennoy. M.: Fizmatlit. (2018).

[10] E. Verlinde, "On the origin of gravity and the laws of Newton", J. High Energy Phys. 4, 1-26 (2011).

[11] T. Padmanabhan, "Thermodynamical Aspects of Gravity: New insights", Rept. Prog. Phys. 73(4), 046901 (44pp) (2010).

[12] L. Susskind, "The World as a hologram”, J. Math. Phys. 36(11), 6377-6396 (1995).

[13] J.D. Bekenstein, "Black Holes and Entropy", Phys. Rev. D. 7(8), 2333-2346. (1975).

[14] S. W. Hawking, "Particle Creation By Black Holes", Commun Math. Phys. 43, 199-220 (1975). 
[15] D.A. Easson, P.H. Frampton, G.F. Smoot, "Entropic accelerating universe", Physics Letters B. 696(3), 273-277 (2011).

[16] T.S. Koivisto, D.F. Mota, M. Zumalacárregui, "Constraining entropic cosmology", J. Cosmol.Astropart. Phys. release 02. id.027 (2011).

[17] Y.S. Myung, "Entropic force and its cosmological implications", Astrophys. Space Sci. 335 (2), 553-559 (2011).

[18] Y.-F. Cai, J. Liu, H. Li, "Entropic cosmology: A unified model of inflation and late-time acceleration", Physics Letters B. 690, 213-219 (2010).

[19] Y.-F. Cai, E. Saridakis, "Inflation in entropic cosmology: Primordial perturbations and non-Gaussianities", Physics Letters B. 697, 280-287 (2011).

[20] T. Qiu, E. N. Saridakis, "Entropic force scenarios and eternal inflation", Phys. Rev. D. 85. 043504 (2012).

[21] S. Basilakos, D. Polarski, J. Sola, "Generalizing the running vacuum energy model and comparing with the entropic-force models", Phys. Rev. D. 86(4), 043010 (2012).

[22] D.A. Easson, P.H. Frampton, G.F. "Smoot, Entropic Inflation", International Journal of Modern Physics A, 27(12) id. 1250066 (2012).

[23] N. Komatsu, S. Kimura, "Entropic cosmology for a generalized black-hole entropy", Physical Review D. 88, 083534 (2013).

[24] N. Komatsu, S. Kimura, "Non-adiabatic-like accelerated expansion of the late universe in entropic cosmology", Phys. Rev. D. 87, 043531 (2013).

[25] N. Komatsu, S. Kimura, "Evolution of the universe in entropic cosmologies via different formulations", Physical Review D. 89(12), 123501 (2014).

[26] N. Komatsu, "Thermodynamic constraints on a varying cosmological-constant-like term from the holographic equipartition law with a power-law corrected entropy", Physical Review D. 96, 103507 (2017)

[27] A. Plastino, M.C. Rocca, "Entropic Forces and Newton's Gravitation". Entropy. 22(3), $273(1-10)(2020)$.

[28] A.D. Wissner-Gross, C.E. Freer, "Causal entropy forces", Phys. Rev. Lett. 110, 168702 (2013).

[29] N.D. Keul, K. Oruganty, E.T.S. Bergman, N.R. Beattie, W.E. McDonald, R. Kadirvelraj, M.L. Gross, R.S. Phillips, S.C. Harvey, Z.A. Wood, "The entropic force generated by intrinsically disordered segments tunes protein function”, Nature. 563, 584-588 (2018).

[30] W. de Sitter, "On the relativity of inertia. Remarks concerning Einstein's latest hypothesis", Proc. Roy. Acad. Sci. (Amsterdam). 19, 1217-1225 (1917).

[31] C. Tsallis, L.J.L. Cirto, "Black hole thermodynamical entropy", Eur. Phys. J. C. 73, id 2487 (2013).

[32] V.G. Czinner, H. Iguchi, "Rényi entropy and the thermodynamic stability of black holes", Phys. Lett. B. 752, 306-310 (2016).

[33] S. Weinberg, "The cosmological constant problem", Reviews of Modern Physics. 61(1), 1-23 (1989).

[34] F.K. Anagnostopoulos, S. Basilakos, E.N. Saridakis, "Observational constraints on Barrow holographic dark energy", Eur. Phys. J. C. 80, 826 (1-9) (2020).

[35] J. D. Barrow, "The area of a rough black hole", Physics Letters B. 808, 135643 (2020).

[36] E.N. Saridakis, "Modified cosmology through spacetime thermodynamics and Barrow horizon entropy", Journal of Cosmology and Astroparticle Physics. 
release 07, article id. 031 (2020).

[37] R. Tolmen, Otnositel'nost', termodinamika i kosmologiya. M.: URSS: Knizhnyy dom «LIBROKOM». (2009).

[38] B. Ryden, Introduction to Cosmology. Cambridge University Press. (2017).

[39] A. Friedmann, "Über die Krümmung des Raumes", Zeitschrift für Physik. 10, 377-386 (1922).

[40] F.K. Anagnostopoulos, S. Basilakos, G. Kofinas, V. Zarikas, "Constraining the Asymptotically Safe Cosmology: cosmic acceleration without dark energy", // Journal of Cosmology and Astroparticle Physics, Issue 02, article id. 053 (2019).

[41] D.F. Torres, H. Vucetich, A. Plastino, "Early Universe Test of Nonextensive Statistics", Phys. Rev. Lett. 79(9), 1588-1590 (1997).

[42] Y. Aditya, S. Mandal, P. Sahoo, D. Reddy, "Observational constraint on interacting Tsallis holographic dark energy in logarithmic Brans-Dicke theory", The European Physical Journal C, 79(12), id. (2019).

[43] G. Wilk, Z. Wlodarczyk, "On the interpretation of nonextensive parameter $q$ in Tsallis statistics and Levy distributions", Phys. Rev. Lett. 84, 2770-2773 (2000).

[44] S. Waheed, "Reconstruction paradigm in a class of extended teleparallel theories using Tsallis holographic dark energy", The European Physical Journal Plus, 135(1), article id.11 (2020).

[45] E.N. Saridakis, S. Basilakos, "The generalized second law of thermodynamics with Barrow entropy", arXiv:2005. 08258 (2020).

[46] S. Basilakos, M. Plionis, J. Sola, "Hubble expansion and structure formation in time varying vacuum models", Phys. Rev. D. 80(8), 083511 (2009).

[47] T. Padmanabhan, S.M. Chitre, "Viscous universes", Physics Letters A, 120(9), 433-436 (1987).

[48] B. Li, J. Barrow, "Does bulk viscosity create a viable unified dark matter model?", Physical Review D, 79(10), id. 103521 (2009).

[49] A. Avelino, U. Nucamendi, "Exploring a matter-dominated model with bulk viscosity to drive the accelerated expansion of the Universe", Journal of Cosmology and Astroparticle Physic. 2010(8), article no. 009 (2010).

[50] X.-H Meng, X. Dou, "Friedmann cosmology with bulk viscosity: a concrete model for dark energy", Communicationsin Theoretical Physics. 52(2), 377-38 (2009).

[51] X. Dou, X.-H. Meng, "Bulk Viscous Cosmology: Unified Dark Matter", Advances in Astronomy, 2011, id.829340 (2011).

[52] O. Gron, "Viscous inflationary universe models", Astrophysics and Space Science. 173, 191-225 (1990).

[53] I. Brevik, O.G. Gorbunova, "Dark energy and viscous cosmology", General Relativity and Gravitation. 37, 2039-2045 (2005).

[54] L. Sebastian, "Dark viscous fluid coupled with dark matter and future singularity", European Physical Journal C. 69, 547-553 (2010).

[55] S. Basilakos, J. Sola, "Entropic-force dark energy reconsidered", Phys. Rev. D. 90(2), 023008 (2014).

[56] A.V. Kolesnichenko, "K postroyeniyu neadditivnoy termodinamiki slozhnykh sistem na osnove statistiki Kurado-Tsallisa", Preprinty IPM im. M.V. Keldysha. 25. 1-40 (2018).

[57] A.V. Kolesnichenko, Statisticheskaya mekhanika i termodinamika Tsallisa neadditivnykh 
system:Vvedenie v teoriyu i prilozheniya. Moskow: LENAND. (Sinergetika ot proshlogo $\mathrm{k}$ budushchemu. № 87), (2019).

[58] A.V. Frolov, L. Kofman, "Inflation and de Sitter thermodynamics", Journal of Cosmology and Astroparticle Physics, Issue 05, article id. 009 (2003).

[59] R.G. Cai, S.P. Kim, "First law of thermodynamics and Friedmann equations of Friedmann-Robertson-Walker universe", Journal of High Energy Physics, Issue 02, id. 050 (2005).

[60] M. Akbar, R.G. Cai, "Thermodynamic Behavior of Friedmann Equations at Apparent Horizon of FRW Universe", Phys. Rev. D. 75, 084003 (2007).

[61] M.V.Keldysh., M.Ya. Marov. Space exploration. Moscow, Science (1981).

[62] A.A. Starobinsky, "A new type of isotropic cosmological models without singularity", Physics Letters B . 91 (1), 99-103 (1980).

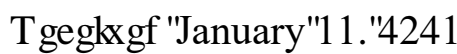

